\title{
Phase II study of erlotinib for previously treated patients with EGFR wild-type non-small-cell lung cancer, following EGFR mutation status reevaluation with the Scorpion Amplified Refractory Mutation System
}

\author{
MASAHIRO MORISE ${ }^{1}$, HIROYUKI TANIGUCHI $^{2}$, HIDEO SAKA $^{3}$, JOE SHINDOH $^{4}$, \\ RYUJIRO SUZUKI $^{5}$, EIJI KOJIMA ${ }^{6}$, TETSUNARI HASE ${ }^{1}$, MASAHIKO ANDO $^{7}$, \\ MASASHI KONDO $^{1}$, HIROSHI SAITO ${ }^{8}$ and YOSHINORI HASEGAWA ${ }^{1}$
}

${ }^{1}$ Department of Respiratory Medicine, Nagoya University Graduate School of Medicine, Nagoya,

Aichi 466-8550; ${ }^{2}$ Department of Respiratory Medicine and Allergy, Tosei General Hospital, Seto,

Aichi 489-8642; ${ }^{3}$ Department of Respiratory Medicine, National Hospital Organization, Nagoya Medical Center, Nagoya, Aichi 460-0001; ${ }^{4}$ Department of Respiratory Medicine, Ogaki Municipal Hospital, Ogaki, Gifu 503-8502;

${ }^{5}$ Department of Respiratory Medicine, Toyohashi Municipal Hospital, Toyohashi, Aichi 441-8085;

${ }^{6}$ Department of Respiratory Medicine, Komaki Municipal Hospital, Komaki, Aichi 485-8520;

${ }^{7}$ Center for Advanced Medicine and Clinical Research, Nagoya University Hospital, Nagoya, Aichi 466-8550; ${ }^{8}$ Department of Respiratory Medicine, Aichi Cancer Center

Aichi Hospital, Okazaki, Aichi 444-0011, Japan

Received January 30, 2014; Accepted June 4, 2014

DOI: $10.3892 / \mathrm{mco} .2014 .354$

\begin{abstract}
While assessing the efficacy of erlotinib in patients with epidermal growth factor receptor (EGFR) wild-type (WT) non-small-cell lung cancer (NSCLC), the sensitivity of the method used for the EGFR mutation analysis may affect the evaluation of the efficacy. We conducted a phase II study of erlotinib for previously treated patients with EGFR WT NSCLC screened by the peptide nucleic acid-locked nucleic acid (PNA-LNA) polymerase chain reaction (PCR) clamp method, which is known to be highly sensitive. The primary endpoint was the objective response rate (ORR). Preplanned reevaluation of the EGFR genotype as an exploratory endpoint was performed using the Scorpion Amplification Refractory Mutation System (S-ARMS) assay. Erlotinib was administered daily until disease progression or development of unacceptable toxicity. A total of 53 evaluable patients were enrolled. The histological subtypes were adenocarcinoma in 40 patients, squamous cell carcinoma in 9 patients and not otherwise specified NSCLC in 4 patients. Partial response (PR) was achieved
\end{abstract}

Correspondence to: Dr Masahiro Morise, Department of Respiratory Medicine, Nagoya University Graduate School of Medicine, 65 Tsurumai-cho, Nagoya, Aichi 466-8550, Japan E-mail: morisem@med.nagoya-u.ac.jp

Key words: erlotinib, non-small-cell lung cancer, epidermal growth factor receptor wild-type in 6 patients ( 4 with adenocarcinoma and 2 with squamous cell carcinoma). The ORR was $11.3 \%$ [95\% confidence interval (CI): 4.3-23.0]. The median progression-free survival (PFS) was 1.8 months (95\% CI: 1.2-2.3). Samples from 26 of the 53 patients $(49.0 \%)$ were available for EGFR mutation reanalysis with the S-ARMS assay. Of these 26 samples, only 1 sample of adenocarcinoma was found to be EGFR mutation-positive (exon 19 deletion) and the patient achieved a PR. The EGFR WT genotype was reconfirmed by the S-ARMS assay in the remaining 25 patients and 2 of these patients exhibited a PR. This study did not meet the primary endpoint, although erlotinib was found to be moderately effective in pretreated patients with EGFR WT NSCLC, even when the EGFR mutational status was confirmed by the highly sensitive PNA-LNA clamp PCR method.

\section{Introduction}

Lung cancer remains the leading cause of cancer-related mortality worldwide. Non-small-cell lung cancer (NSCLC) is the predominant histological type of lung cancer and $\sim 70.0 \%$ of all NSCLC patients have advanced-stage IIIB or IV disease at diagnosis. Platinum-based chemotherapy is currently the standard treatment for advanced NSCLC; however, almost all the patients treated by initial chemotherapy alone eventually develop a relapse.

Erlotinib, a selective epidermal growth factor receptor (EGFR)-tyrosine kinase inhibitor (TKI), is currently recommended as second- or third-line standard treatment in patients with NSCLC (1). The presence of activating somatic 
mutations in the EGFR gene has been shown to be a predictor of the response to treatment with EGFR-TKIs (2) and first-line EGFR-TKI therapy for patients with EGFR mutation-positive NSCLC was shown to improve the progression-free survival (PFS) compared to standard platinum-based chemotherapy (3-6). However, the results of subgroup analyses in the BR21 and SATURN trials suggest that erlotinib may also be beneficial to patients with EGFR wild-type (WT) NSCLC $(1,7)$.

While assessing the efficacy of erlotinib in patients with EGFR WT NSCLC, the sensitivity of the method(s) used for the EGFR mutation analysis may affect the results of the evaluation. Although direct DNA sequencing has been widely used for EGFR mutation analysis, several new techniques, such as the peptide nucleic acid-locked nucleic acid (PNA-LNA) polymerase chain reaction (PCR) clamp method and the Scorpion Amplification Refractory Mutation System (S-ARMS) assay are currently available $(8,9)$. Kim et al $(10)$ reported a higher sensitivity of the PNA-LNA clamp method as compared to direct DNA sequencing for the detection of EGFR mutations in patients with NSCLC. In their study, the EGFR mutation positivity rate in 240 NSCLC patients was $34.6 \%$ when assessed by the PNA-LNA clamp method, but only $26.3 \%$ when assessed by direct DNA sequencing. Therefore, it is possible that erlotinib is found to be considerably less effective in patients with EGFR WT NSCLC, when the EGFR genotype is confirmed by highly sensitive methods, such as the PNA-LNA clamp method.

In addition, the predictive value of KRAS mutations for the efficacy of erlotinib in patients with EGFR WT NSCLC has not been fully elucidated. It was previously suggested that the presence of KRAS mutations may predict a poor response to EGFR-TKI therapy in patients with NSCLC (11). However, the EGFR mutation status may be a confounding factor in the analysis of the predictive value of KRAS mutations, since KRAS and EGFR mutations exhibit a strong negative correlation and EGFR mutation is a predictor of the response to EGFR-TKI therapy. Therefore, further evaluation of the predictive value of KRAS mutations in patients with EGFR WT NSCLC is required.

Based on these findings, we conducted a multicenter phase II trial of erlotinib for previously treated patients with EGFR WT NSCLC. The primary endpoint of this study was to assess the efficacy and safety of erlotinib in patients with EGFR WT NSCLC, as confirmed by the PNA-LNA clamp method, which is a highly sensitive method for EGFR mutation analysis. Preplanned reevaluation of the EGFR and KRAS mutation status as exploratory endpoints was performed using the S-ARMS assay in this study.

\section{Patients and methods}

Study design. This study was a multicenter, open-label, single-arm, phase II trial conducted in Japan. The study protocol was approved by the Central Japan Lung Study Group (CJLSG) Protocol Review Committee and the Institutional Review Board of each center as the CJLSG 0903 trial. The study was performed in accordance with the principles laid out in the Declaration of Helsinki and is registered with the University Hospital Medical Information Network in Japan (no. 000002692). The primary endpoint was the objective response rate (ORR) and the secondary endpoints were disease control rate (DCR), PFS, overall survival (OS) and safety. Moreover, if residual samples were available, we performed a preplanned reevaluation of the EGFR mutation status and KRAS mutation analysis with the S-ARMS assay as a secondary endpoint.

Eligibility criteria. Pretreated stage IIIB/IV NSCLC patients were assessed regarding their eligibility for enrollment in this study. The main inclusion criteria were as follows: Pathologically proven NSCLC; EGFR WT genotype confirmed by the PNA-LNA PCR clamp method; history of one or two prior chemotherapies, including at least one platinum-based chemotherapy; age $\geq 20$ years; Eastern Cooperative Oncology Group performance status (PS) of 0-2; adequate bone marrow, hepatic and renal function; at least one measurable lesion as defined by the Response Evaluation Criteria in Solid Tumors (RECIST), version 1.1 (12); life expectancy of $\geq 3$ months; and patient willingness to provide written informed consent. The main exclusion criteria were as follows: Pulmonary disorders, such as interstitial lung disease, pneumoconioses, or active radiation pneumonitis; severe eye disorders; and massive pleural or pericardial effusion.

EGFR genotype testing for eligibility. The PNA-LNA PCR clamp method was used for confirmation of the EGFR mutation status in the NSCLC patients prior to enrollment. This method is a highly sensitive and simple procedure for the detection of 13 known EGFR mutations (8). For this study, we enrolled patients with the WT allele of EGFR in all 13 mutation sites. A total of 5 tissue slides $(5-\mu \mathrm{m})$ or pleural effusion cytology samples containing tumor cells were used for the analysis. Tissue slides were prepared from tumor cell-rich sections of formalin-fixed paraffin-embedded tumor samples. In Japan, the PNA-LNA PCR clamp method is commercially available and performed by the Mitsubishi Chemical Medience Corporation (Tokyo, Japan).

Screening of tumors for the KRAS genotype and reanalysis of the EGFR mutation status using the S-ARMS assay. Following completion of patient enrollment, the tumor samples available for KRAS mutation analysis and EGFR mutation reanalysis were collected. DNA was extracted at the laboratory of the Department of Respiratory Medicine, Nagoya University Graduate School of Medicine, using the QIAamp DNA Mini kit (Qiagen, Tokyo, Japan), followed by quantitation of the DNA. According to a previous report, the PNA-LNA PCR clamp method and the S-ARMS assay exhibit an equally high sensitivity for the detection of the EGFR mutation status (13). Therefore, we prioritized KRAS mutation screening if the amount of DNA available was not sufficient for evaluation of both the KRAS and EGFR mutation status by the S-ARMS assay. S-ARMS analysis for the detection of EGFR mutation was performed using the EGFR Mutation RGQ PCR kit (Qiagen, Manchester, UK) and S-ARMS analysis for evaluation of the KRAS mutation status was performed using the KRAS PCR kit (Qiagen, Manchester), which is able to detect 7 mutations in codons 12 and 13 of the KRAS gene. 
Treatment. Oral erlotinib was administered at a dose of $150 \mathrm{mg}$ daily until disease progression or development of unacceptable toxicity. The erlotinib dose was reduced (first reduction to $100 \mathrm{mg}$ daily and second reduction to $50 \mathrm{mg}$ daily) or treatment was interrupted in the event of any grade 3 non-hematological toxicity. Dose escalation was not permitted. In the event of development of interstitial lung disease (ILD) of any grade or any grade 4 toxicity, the protocol was discontinued.

Efficacy and safety evaluation. Tumor response was assessed in accordance with RECIST, version 1.1 (12). The baseline assessment included chest and upper abdominal computed tomography (CT), head $\mathrm{CT}$ or magnetic resonance imaging and bone scintigraphy or ${ }^{18} \mathrm{~F}$-fluorodeoxyglucose-positron emission tomography. Assessment of the tumor response was performed every 4 weeks during the first 8 weeks, every 8 weeks during the subsequent 40 weeks and every 12 weeks thereafter. In this study, the definition of stable disease (SD) required a duration of $\geq 8$ weeks. PFS was defined as the time from the date of study enrollment until the date of objectively determined progressive disease (PD) or death due to any cause or the date of the last follow-up. OS was defined as the time from the date of study enrollment until death due to any cause or the date of last follow-up. Toxicity was evaluated using the Common Toxicity Criteria for Adverse Events (version 3.0).

Statistical analysis. The primary endpoint was the ORR and the sample size for the trial was calculated using Simon's two-stage design. Assuming that a response rate of $18.0 \%$ indicates potential usefulness, while a rate of $6.8 \%$ is the lower limit of interest, with $\alpha=0.05$ and $\beta=0.20$, the estimated accrual number was 49 patients. In this study, the rate of the lower limit of interest was adopted based on the ORRs of docetaxel reported in previous phase III studies $(14,15)$. Among these, $\geq 7$ responders were required for this therapy to be considered worthy of further evaluation. We selected a target sample number of 54, to allow for 5 dropouts. The differences in ORR according to histology were analyzed using the Mantel extension test adjusted for PS and M factor (M0, M1a and M1b). A stratified log-rank test adjusted for these factors was used to evaluate the difference in PFS according to histology. $\mathrm{P}<0.05$ was considered to indicate a statistically significant difference.

\section{Results}

Patient characteristics. Between February, 2010 and April, 2012, a total of 55 patients were enrolled. A review of the data indicated that 2 of the patients enrolled in this study did not fulfill the eligibility criteria listed in the study protocol and the remaining 53 patients were included in the analysis as evaluable. The characteristics of the 53 patients are summarized in Table I. The median age of the patients was 67 years (range, 47-77 years). The histological subtypes were non-squamous cell carcinoma (non-SCC) in 44 patients [adenocarcinoma, 40 patients; and not otherwise specified (NOS), 4 patients] and SCC in 9 patients. The number of prior chemotherapies was 1 in 26 patients $(49.0 \%)$ and 2 in the remaining 27 patients $(51.0 \%)$.
Table I. Patient characteristics.

\begin{tabular}{|c|c|}
\hline Characteristics & $\begin{array}{l}\text { Patient no. }(\%) \\
\qquad(\mathrm{n}=53)\end{array}$ \\
\hline \multicolumn{2}{|l|}{ Age, years } \\
\hline Median & 67 \\
\hline Range & $47-77$ \\
\hline \multicolumn{2}{|l|}{ Gender } \\
\hline Male & $43(81.0)$ \\
\hline Female & $10(19.0)$ \\
\hline \multicolumn{2}{|l|}{ Smoking status } \\
\hline Never & $7(13.0)$ \\
\hline Former/current & $46(87.0)$ \\
\hline \multicolumn{2}{|l|}{ Histology } \\
\hline Adenocarcinoma & $40(75.0)$ \\
\hline Squamous cell carcinoma & $9(17.0)$ \\
\hline NOS & $4(8.0)$ \\
\hline \multicolumn{2}{|l|}{ No. of prior chemotherapies } \\
\hline 1 & $26(49.0)$ \\
\hline 2 & $27(51.0)$ \\
\hline \multicolumn{2}{|l|}{ Stage } \\
\hline IIIB & $2(4.0)$ \\
\hline \multicolumn{2}{|l|}{ IV } \\
\hline M1a & $16(30.0)$ \\
\hline M1b & $35(66.0)$ \\
\hline \multicolumn{2}{|l|}{ ECOG PS } \\
\hline 0 & $23(43.4)$ \\
\hline 1 & $24(45.3)$ \\
\hline 2 & $6(11.3)$ \\
\hline
\end{tabular}

NOS, not otherwise specified; ECOG PS, Eastern Cooperative Oncology Group performance status.

Efficacy. The median treatment duration was 51 days (range, 5-404 days). Of the 53 eligible patients, partial response (PR) was obtained in 6 patients (4 with adenocarcinoma and 2 with SCC), yielding an ORR of $11.3 \%$ (95\% confidence interval (CI): 4.3-23.0). SD was observed in 9 patients and the DCR was $28.3 \%$ (95\% CI: 16.8-42.3). The ORR according to the histology was $9.1 \%$ (95\% CI: 2.5-21.7) in patients with non-SCC and 22.2\% (95\% CI: 2.8-60.0) in patients with SCC. The difference in the ORR between these two groups was not statistically significant ( $\mathrm{P}=0.29$, Mantel extension test). A summary of the tumor responses is provided in Table II. At the time of the analysis, 48 patients $(91.0 \%)$ had developed disease progression and $34(64.0 \%)$ had succumbed to the disease. The median PFS of the entire patient cohort was 1.8 months (95\% CI: 1.2-2.3). The median PFS in the patients with non-SCC and SCC was 1.7 months (95\% CI: 1.2-2.1), and 2.2 months (95\% CI: 1.0-11.3), respectively, without a statistically significant difference $(\mathrm{P}=0.54$, stratified log-rank test). The Kaplan-Meier survival curve for PFS is shown in Fig. 1. The median OS was 6.4 months (95\% CI: 4.5-10.4) and the 
Table II. Tumor response.

\begin{tabular}{lccc}
\hline $\begin{array}{l}\text { Type of } \\
\text { response }\end{array}$ & $\begin{array}{c}\text { Total } \\
(\mathrm{n}=53)\end{array}$ & $\begin{array}{c}\text { Non-SCC } \\
(\mathrm{n}=44)\end{array}$ & $\begin{array}{c}\text { SCC } \\
(\mathrm{n}=9)\end{array}$ \\
\hline $\mathrm{CR}$ & 0 & 0 & 0 \\
$\mathrm{PR}$ & 6 & 4 & 2 \\
$\mathrm{SD}$ & 9 & 7 & 2 \\
$\mathrm{PD}$ & 37 & 32 & 5 \\
$\mathrm{NE}$ & 1 & 1 & 0 \\
ORR, \% & 11.3 & 9.1 & 22.2 \\
$(95 \% \mathrm{CI})$ & $(4.3-23.0)$ & $(2.5-21.7)$ & $(2.8-60.0)$ \\
DCR, \% & 28.3 & 25.0 & 44.4 \\
$(95 \% \mathrm{CI})$ & $(16.8-42.3)$ & $(13.2-40.3)$ & $(13.7-78.8)$ \\
\hline
\end{tabular}

SCC, squamous cell carcinoma; non-SCC, adenocarcinoma and not otherwise specified non-small-cell lung cancer; CR, complete response; $\mathrm{PR}$, partial response; $\mathrm{SD}$, stable disease (a duration of $\geq 8$ weeks was required for the definition of SD in this study); PD, progressive disease; NE, not evaluable; ORR, objective response rate; CI, confidence interval; DCR, disease control rate.

Table III. Adverse events in the patients $(n=53)$.

\begin{tabular}{lccccc}
\hline \multirow{2}{*}{$\begin{array}{l}\text { Adverse } \\
\text { events }\end{array}$} & \multicolumn{3}{c}{ Grade (patient no.) } & $\begin{array}{c}\text { \% of patients } \\
\text { with grade 3-4 } \\
\text { toxicity }\end{array}$ \\
\cline { 2 - 5 } Skin rash & 1 & 2 & 3 & 4 & 11.3 \\
Diarrhea & 11 & 26 & 6 & 0 & 1.9 \\
Anorexia & 16 & 1 & 1 & 0 & 9.4 \\
Nausea & 14 & 6 & 5 & 0 & 0.0 \\
Vomiting & 4 & 2 & 0 & 0 & 1.9 \\
Fatigue & 1 & 1 & 1 & 0 & 5.7 \\
Stomatitis & 8 & 7 & 3 & 0 & 1.9 \\
Ocular disorders & 2 & 0 & 1 & 0 & 1.9 \\
ALT increased & 7 & 4 & 1 & 2 & 5.7 \\
AST increased & 10 & 4 & 1 & 2 & 5.7 \\
Amy increased & 0 & 1 & 0 & 1 & 1.9 \\
Leukopenia & 1 & 1 & 0 & 0 & 0.0 \\
Thrombocytopenia & 6 & 0 & 0 & 0 & 0.0 \\
ILD & 2 & 0 & 1 & 0 & $5.6^{\mathrm{a}}(\mathrm{G} 3-5)$
\end{tabular}

${ }^{a}$ Grade 5 ILD was observed in 2 patients. ALT, alanine transaminase; AST, aspartate transaminase; amy, amylase; ILD, interstitial lung disease.

Kaplan-Meier survival curve for OS is shown in Fig. 2. The median OS in the patients with 1 and 2 prior chemotherapies was 8.5 and 5.5 months, respectively.

Safety. The adverse events are summarized in Table III. The major adverse events were rash in $81.1 \%$ of the patients $(11.3 \% \geq$ grade 3$)$ and anorexia in $47.1 \%$ (9.4\% $\geq$ grade 3$)$. No grade 3 or 4 hematological adverse events were observed. Grade 3-5 ILD was reported in 3 patients (5.6\%) and grade 5
A

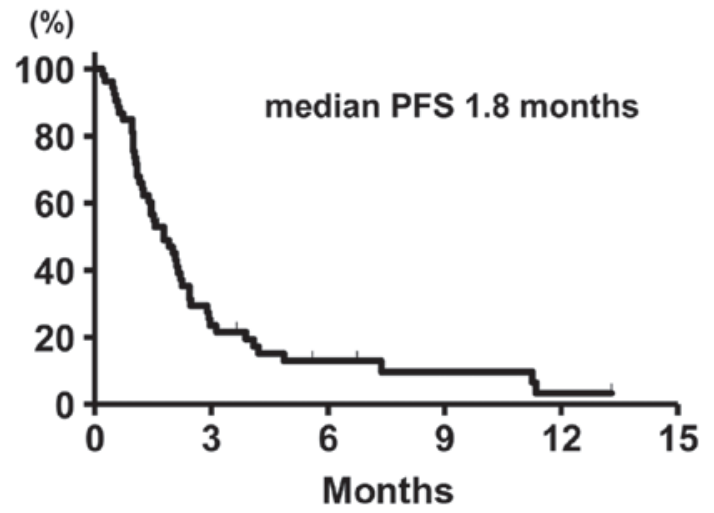

B

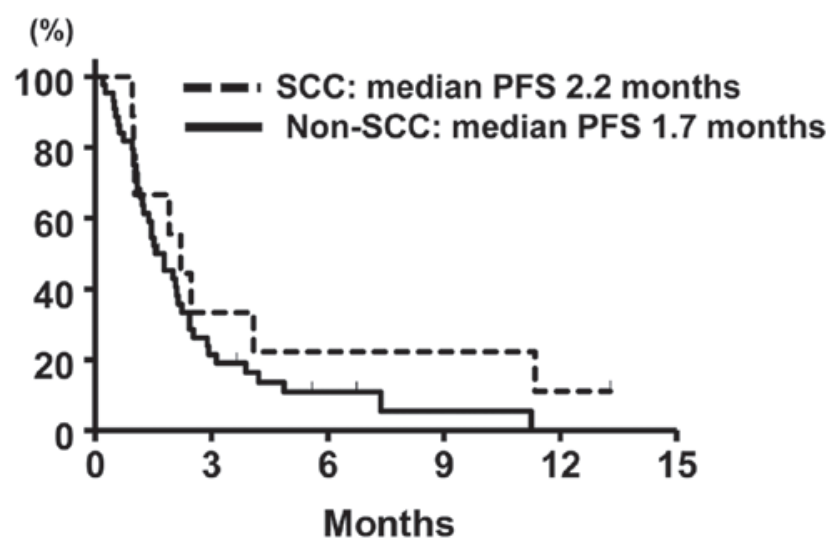

Figure 1. Kaplan-Meier survival curves for (A) progression-free survival (PFS) in the overall study population $(\mathrm{n}=53)$ and (B) progression-free survival in subgroups classified according to histology [squamous cell carcinoma (SCC) $(n=9)$ vs. non-SCC $(n=44)]$.

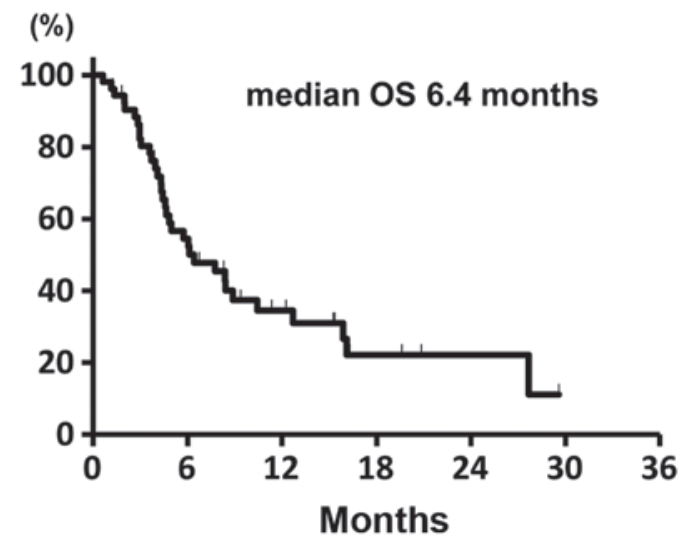

Figure 2. Kaplan-Meier survival curves for overall survival (OS ) of the overall study population $(\mathrm{n}=53)$.

ILD possibly related to erlotinib in 2 patients (3.8\%). In the 2 patients with grade 5 ILD, the baseline chest CT revealed carcinomatous lymphangitis and lung cancer progression was concurrently detected by chest $\mathrm{CT}$ at the time of development of the ILD.

EGFR mutation reanalysis with the S-ARMS assay and KRAS mutation screening. Samples from 26 patients (49\% of the 
Table IV. KRAS mutation-positive patients.

\begin{tabular}{lccccc}
\hline Case & Gender & Smoking status & Smoking index & Amino acid change & Best overall response \\
\hline 1 & Male & Former & 1020 & Gly12Ala (GGT>GCT) & PD \\
2 & Male & Current & 1020 & Gly12Cys (GGT>TGT) & PD \\
3 & Male & Current & 1000 & Gly12Ala (GGT>GCT) & PD \\
4 & Male & Former & 1520 & Gly12Cys (GGT>TGT) & PD \\
\hline
\end{tabular}

Gly, glycine; Ala, alanine; Cys, cysteine; PD, progressive disease.

eligible patients) were available for EGFR mutation reanalysis. Of these, only 1 patient with adenocarcinoma was found to be EGFR mutation-positive (exon 19 deletion) NSCLC with the S-ARMS assay and this patient exhibited a PR. In the remaining 25 patients, EGFR WT was reconfirmed by the S-ARMS assay and two of these patients exhibited a PR. The ORR was $8.0 \%$ in the NSCLC patients with EGFR WT as confirmed by both the PNA-LNA PCR clamp method and the S-ARMS assay.

The KRAS mutation status was screened by the S-ARMS assay in samples obtained from 44 patients, of which DNA amplification was unsuccessful in 2. KRAS mutation screening was successfully performed in the samples from the remaining 42 patients ( $79.0 \%$ of eligible patients). Of these 42 patients, $4(9.1 \%)$ were found to be KRAS mutation-positive. The characteristics of these 4 patients and the sites of the KRAS mutations are listed in Table IV. As regards treatment response, PD was observed in all 4 patients. By contrast, the ORR and median PFS in the patients with KRAS WT NSCLC were $6.9 \%$ and 1.9 months, respectively.

\section{Discussion}

In this study, we evaluated the efficacy and safety of erlotinib in pretreated patients with NSCLC harboring EGFR WT as confirmed by the PNA-LNA clamp method, which is reported as being highly sensitive. This study did not meet the primary endpoint based on the reported ORRs of docetaxel in previous studies, although erlotinib treatment was associated with an ORR of $11.3 \%$.

Two recent phase III studies reported the inferiority of erlotinib compared to docetaxel regarding ORR and PFS in EGFR WT NSCLC patients $(16,17)$. Based on these results, including the findings of our study, it appears that docetaxel should be preferred as second-line therapy, if not used as a part of first-line platinum based combination therapy.

However, there remains the clinical question of whether erlotinib should not be used for EGFR WT NSCLC in any-line setting. In our opinion, erlotinib monotherapy may be an viable option in pretreated patients with EGFR WT NSCLC following failure of docetaxel treatment for the following reasons: First, EGFR WT was reconfirmed by the S-ARMS assay in 25 of the 26 patient samples examined in this study, of which $2(8.0 \%)$ achieved a PR. Our results suggested that erlotinib may still be effective against EGFR WT NSCLC, even when the EGFR mutation status is confirmed by two different highly sensitive methods.
Second, a discordance in the EGFR mutation status between the PNA-LNA clamp method and S-ARMS assay was observed in 1 patient in this study. Although large, tumor cell-rich samples are required for accurate EGFR mutation analysis, we cannot, in general, obtain surgically resected specimens from advanced NSCLC patients in clinical practice. Fukui et al (18) verified the accuracy of the EGFR mutation analysis in small samples by high-resolution melting analysis, which has also been reported to be a highly sensitive method. In that study, the results of DNA sequencing combined with laser capture microdissection in paired surgically resected specimens revealed a few false-negative results in small samples. Those data suggested that it may be difficult to determine the EGFR mutation status with complete accuracy in small tissue samples, irrespective of the sensitivity of the method used. Therefore, if we do not use erlotinib for EGFR WT NSCLC in any-line setting, we may miss the opportunity to attempt elrotinib treatment for patients with a false-negative EGFR mutation result. This may also lead to loss of the significant survival benefit obtained from EGFR-TKI therapy for EGFR mutation-positive NSCLCs.

We succeeded in obtaining 42 samples (79\% of the eligible patients) for KRAS mutation screening. KRAS mutations were detected in 4 of the 42 patients screened $(9.5 \%)$ and all the KRAS mutation-positive patients exhibited PD. In a phase III study conducted to compare erlotinib and pemetrexed, none of the patients with KRAS mutation-positive NSCLC responded to erlotinib treatment, which was similar to the findings of our study (19). These results should be interpreted with caution, as we could not exclude the KRAS mutation status as a potential prognostic factor. However, the presence of KRAS mutation may be useful as a negative predictive factor, at least regarding response to erlotinib therapy, in patients with EGFR WT NSCLC.

We performed a subgroup analysis according to histological subtype. In patients with EGFR mutated NSCLC, the efficacy of EGFR-TKIs for SCC appeared to be lower compared to that for non-SCC (20). However, SCC histology may not be associated with poor efficacy of erlotinib in patients with EGFR WT NSCLC based on our results. Molecular biomarkers, such as KRAS, may be required to select suitable candidates for erlotinib treatment among patients with EGFR WT NSCLC.

The toxicity profile of erlotinib in this study, in terms of the incidence/grade of skin rash, diarrhea and hematological toxicities, was consistent with previous reports. However, grade 3-5 ILD was reported in 3 patients (5.8\%). In a large-scale surveillance study conducted in Japan, the incidence of ILD 
was also higher compared to that reported by the BR21 and SATURN trials $(1,7,21)$. Further studies are required to determine whether there are ethnic differences in the incidence of ILD, as suggested by a previous study (22).

In conclusion, this study did not meet the primary endpoint, although erlotinib was found to be moderately effective in pre-treated patients with EGFR WT NSCLC, even when the EGFR mutational status was confirmed by the highly sensitive PNA-LNA clamp PCR method.

\section{Acknowledgements}

This study was supported by the Central Japan Lung Study Group (CJLSG), a non-profit organization supported by unrestricted donations from the following pharmaceutical companies: Chugai Pharmaceutical Co., Ltd. (Tokyo, Japan); Shionogi \& Co., Ltd. (Osaka, Japan); Daiichi Sankyo Co., Ltd. (Tokyo, Japan); Dainippon Sumitomo Pharma Co., Ltd.(Osaka, Japan); Janssen Pharmaceutical K.K. (Tokyo, Japan); Eli Lilly Japan K.K. (Kobe, Japan); Taisho Toyama Pharmaceutical Co., Ltd.; Meiji Seika Pharma Co., Ltd.; MSD K.K.; Bayer Holding Ltd.; Astellas Pharma Inc. and Nippon Boehringer Ingelheim Co., Ltd. (all from Tokyo, Japan). Dr Morise reported receiving honoraria for lecturing from Chugai Pharmaceutical Co.; Dr Taniguchi has served as a member of the advisory boards at Chugai Pharmaceutical Co., Boehringer Ingelheim and Shionogi \& Co., Ltd.; Dr Saka reported receiving two grants from Chugai Pharmaceutical Co., which were paid to Nagoya Medical Center (Nagoya, Japan); Dr Hase reported receiving honoraria for lecturing from Chugai Pharmaceutical Co., Pfizer Inc. (New York City, NY, USA) and Astra Zeneca Co. (London, UK); Dr Ando reported having a board membership at Chugai Pharmaceutical Co.; Dr Kondo reported receiving honoraria for lecturing from Chugai Pharmaceutical Co.; Dr Saito received research funding from Chugai Pharmaceutical Co.; Dr. Hasegawa reported receiving honoraria for lecturing from Chugai Pharmaceutical Co. and receiving a grant from Chugai-Pharmaceutical Co. that was paid to Nagoya University

\section{References}

1. Shepherd FA, Rodrigues Pereira J, et al; National Cancer Institute of Canada Clinical Trials Group: Erlotinib in previously treated non-small-cell lung cancer. N Engl J Med 353: 123-132, 2005.

2. Paez JG, Janne PA, Lee JC, et al: EGFR mutations in lung cancer: correlation with clinical response to gefitinib therapy. Science 304: 1497-1500, 2004.

3. Maemondo M, Inoue A, Kobayashi K, et al: Gefitinib or chemotherapy for non-small-cell lung cancer with mutated EGFR. N Engl J Med 362: 2380-2388, 2010.

4. Mitsudomi T, Morita S, Yatabe Y, et al: Gefitinib versus cisplatin plus docetaxel in patients with non-small-cell lung cancer harbouring mutations of the epidermal growth factor receptor (WJTOG3405): an open label, randomised phase 3 trial. Lancet Oncol 11: 121-128, 2010.

5. Zhou C, Wu YL, Chen G, et al: Erlotinib versus chemotherapy as first-line treatment for patients with advanced EGFR mutation-positive non-small-cell lung cancer (OPTIMAL, CTONG-0802): a multicentre, open-label, randomised, phase 3 study. Lancet Oncol 12: 735-742, 2011.

6. Rosell R, Carcereny E, Gervais R, et al: Erlotinib versus standard chemotherapy as first-line treatment for European patients with advanced EGFR mutation-positive non-small-cell lung cancer (EURTAC): a multicentre, open-label, randomised phase 3 trial. Lancet Oncol 13: 239-246, 2012.
7. Cappuzzo F, Ciuleanu T, Stelmakh L, et al: Erlotinib as maintenance treatment in advanced non-small-cell lung cancer: a multicentre, randomised, placebo-controlled phase 3 study. Lancet Oncol 11: 521-529, 2010.

8. Nagai Y, Miyazawa H, Huqun, et al: Genetic heterogeneity of the epidermal growth factor receptor in non-small cell lung cancer cell lines revealed by a rapid and sensitive detection system, the peptide nucleic acid-locked nucleic acid PCR clamp. Cancer Res 65: 7276-7282, 2005.

9. Kimura H, Fujiwara Y, Sone T, et al: High sensitivity detection of epidermal growth factor receptor mutations in the pleural effusion of non-small cell lung cancer patients. Cancer Sci 97: 642-648, 2006.

10. Kim HJ, Lee KY, Kim YC, et al: Detection and comparison of peptide nucleic acid-mediated real-time polymerase chain reaction clamping and direct gene sequencing for epidermal growth factor receptor mutations in patients with non-small cell lung cancer. Lung Cancer 75: 321-325, 2012.

11. Linardou H, Dahabreh IJ, Kanaloupiti D, et al: Assessment of somatic k-RAS mutations as a mechanism associated with resistance to EGFR-targeted agents: a systematic review and meta-analysis of studies in advanced non-small-cell lung cancer and metastatic colorectal cancer. Lancet Oncol 9: 962-972, 2008.

12. Eisenhauer EA, Therasse P, Bogaerts J, et al: New response evaluation criteria in solid tumours: revised RECIST guideline (version 1.1). Eur J Cancer 45: 228-247, 2009.

13. Goto K, Satouchi M, Ishii G, et al: An evaluation study of EGFR mutation tests utilized for non-small-cell lung cancer in the diagnostic setting. Ann Oncol 23: 2914-2919, 2012.

14. Fossella FV, DeVore R, Kerr RN, et al: Randomized phase III trial of docetaxel versus vinorelbine or ifosfamide in patients with advanced non-small-cell lung cancer previously treated with platinum-containing chemotherapy regimens. The TAX 320 Non-Small Cell Lung Cancer Study Group. J Clin Oncol 18: 2354-2362, 2000.

15. Shepherd FA, Dancey J, Ramlau R, et al: Prospective randomized trial of docetaxel versus best supportive care in patients with non-small-cell lung cancer previously treated with platinum-based chemotherapy. J Clin Oncol 18: 2095-2103, 2000.

16. Garassino MC, Martelli O, Broggini M, et al; TAILOR trialists: Erlotinib versus docetaxel as second-line treatment of patients with advanced non-small-cell lung cancer and wild-type EGFR tumours (TAILOR): a randomised controlled trial. Lancet Oncol 14: 981-988, 2013.

17. Kawaguchi T, Ando M, Asami K, et al: Randomized phase III trial of erlotinib versus docetaxel as second- or third-line therapy in patients with advanced non-small cell lung cancer: Docetaxel and Erlotinib Lung Cancer Trial (DELTA). J Clin Oncol: May 19, 2014 (Epub ahead of print).

18. Fukui T, Ohe Y, Tsuta K, et al: Prospective study of the accuracy of EGFR mutational analysis by high-resolution melting analysis in small samples obtained from patients with non-small cell lung cancer. Clin Cancer Res 14: 4751-4757, 2008.

19. Karampeazis A, Voutsina A, Souglakos J, et al: Pemetrexed versus erlotinib in pretreated patients with advanced non-small cell lung cancer: a Hellenic Oncology Research Group (HORG) randomized phase 3 study. Cancer 119: 2754-2764, 2013.

20. Hata A, Katakami N, Yoshioka H, et al: How sensitive are epidermal growth factor receptor-tyrosine kinase inhibitors for squamous cell carcinoma of the lung harboring EGFR gene-sensitive mutations? J Thorac Oncol 8: 89-95, 2013.

21. Nakagawa K, Kudoh S, Ohe Y, et al: Postmarketing surveillance study of erlotinib in Japanese patients with non-small-cell lung cancer (NSCLC): an interim analysis of 3488 patients (POLARSTAR). J Thorac Oncol 7: 1296-1303, 2012.

22. Yoshioka H, Hotta K, Kiura K, et al; Okayama Lung Cancer Study Group: A phase II trial of erlotinib monotherapy in pretreated patients with advanced non-small cell lung cancer who do not possess active EGFR mutations: Okayama Lung Cancer Study Group trial 0705. J Thorac Oncol 5: 99-104, 2010. 